\title{
Local Electric Polarization Vector Detection
}

\author{
Kwang Geol Lee and DaiSik Kim \\ Seoul National University \\ Republic of Korea
}

\section{Introduction}

The function of nanophotonic devices, such as metamaterials for the visible range, photonic and plasmonic crystals, nanoscale waveguides, resonators, switches or optical antennas relies essentially on our ability to tailor and control electromagnetic fields on a subwavelength scale, in much the same way as the wavefunction of electronic nanostructures is localized on an atomic scale. Unlike matter waves, these localized electromagnetic waves are vectorial in nature, and their orientation and magnitude varies on a sub-wavelength scale. Therefore it is vital for a complete description of light in nano-scale devices to map the field vectors with subwavelength resolution. Experimental techniques aimed at probing the field intensity, e.g. aperture-less or aperture-based near-field microscopies, are rapidly improving, but the local orientation of the electromagnetic polarization vector, a fundamental property of the local electromagnetic field, could not be accessed experimentally.

Yet, the orientation of the field vector is a key quantity in many thousands of theoretical studies on nano-optics, nanophotonic devices and optical sciences in general. Evidently, the ability to experimentally probe electromagnetic field vectors with nanometer resolution is of fundamental importance for understanding and improving nano-optical devices and for reconciling experiment and theory in the bourgeoning field of nanophotonics.

In this chapter, we describe and demonstrate the local field polarization vector detection using the gold nanoparticle (GNP) attached tip as the local field scatterer acting as a nanometer-scaled polarizer. For a suitably small GNP, the far-field scattering is dominated by the electric dipole radiation. Dipole radiation conserve its polarization state into the farfield region enabling characterization of the dipole moment induced at the GNP by measuring the far-field polarization state. And also the dipole moment is determined by the local electric field via polarizability tensor of GNP. Therefore, by characterizing the polarizability tensor of GNP and the polarization state of far-field scattered light, the local electric field vector can be reconstructed. In doing that, the different scattering shape of GNP, the polarizability tensor, is carefully measured and considered to get a consistant result independently of the tip shape.

Mapping the local polarization vector in the near-field demands a careful consideration of the surface effect. For example, far field detected light is a complex mixture of the scattered light from GNP and its reflected light at the sample surface which interfere each other strongly depending on the polarization. This so called image dipole effect should be taken into account to correctly address the reconstructed local field vectors in near-field region. In the last section of this chaper, we discuss the limitations of our method and give suggestions to improve the functionality of the polarization vector microscopy.

Source: Recent Optical and Photonic Technologies, Book edited by: Ki Young Kim, ISBN 978-953-7619-71-8, pp. 450, January 2010, INTECH, Croatia, downloaded from SCIYO.COM 


\section{Local field polarization vector detection on nanoscale}

\subsection{Polarizability tensor measurement of GNP attached tips}

The metal particle has been used in a wide range of applications owing to its good photostability. For example, GNP is one of the excellent candidates as a tracking label in biosensing. It also can serve as an optical antenna to enhance the spontaneous emission rate of single molecules by using its plasmon resonance (Kühn et al., 2006). Moreover, a small GNP, less than $100 \mathrm{~nm}$ in diameter, is a good dipolar scatterer and it could be used as a nano-sized polarizer to detect the local electric field polarization vectors (Lee et al., 2007a). In the reconstruction of the local field vectors from the far-field detected signal radiated by the GNP, it is crucial to correctly and efficiently measure the polarizability tensor of the GNP. In this section, we present theoretically and experimentally how the polarizability tensor can be read out from two types of scattering measurement - rotational polarizer analyzer ellipsometry (RPAE) and rotational polarizer ellipsometry (RPE). By comparing two methods, the pros and cons of each method are discussed. Finally, we show the experimental results validating the model and the data process.
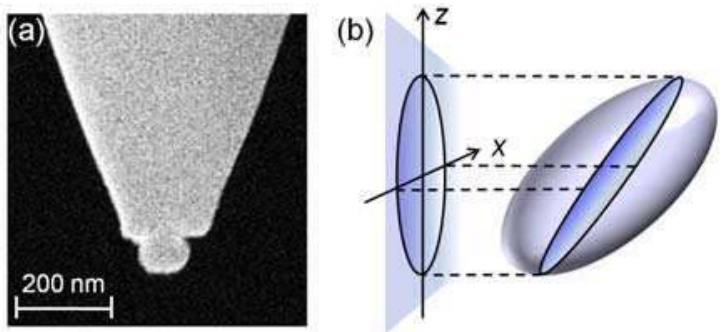

Fig. 1. (a) An SEM picture of a GNP attached tapered glass tip. (b) The projection of an arbitrary ellipsoid onto a plane makes an ellipse. In the same analogy, the geometrical shape of the polarizability tensor of an ellipsoidal GNP in a 2-dimensional plane can be regarded as an ellipse.

The GNP attached tips (Kalkbrenner et al., 2001) are prepared as following. Sharp glass fiber tips with the tip apex size of 100 200 $\mathrm{nm}$ are made by the chemical etching (hydrofluoric acid, $48 \%, 65$ mins) or by using a mechanical puller machine. Then tips are chemically treated to generate a chemical layer sticky to the GNP. For the case of APTES (3aminopropyltriethoxysilane), GNP is bonded to the glass fiber through the electro-static force, on the other hand, APTMS (3-aminopropyltrimethoxysilane) forms a layer which makes a covalent bonding to the GNP (Keating et al., 1999). This surface treated tip is scanned onto a cover slip or a slide glass where the GNP colloid is spin-coated on it. When the tip moves across a GNP, the GNP eventually sticks to the tip and moves together. A scanning electron microscope (SEM) image of the tip attached a $100 \mathrm{~nm}$ diameter sized GNP is shown in Fig. 1(a). The size variation of GNPs in the colloid is less 8\% (from data sheet of BBinternational) and there is a small variation in shape also. Additional to these intrinsic morphological distributions, the attaching process may generate a deformation of the particle shape due to the squeezing forces applied between the tip and the sample surface. Therefore the geometrical shape of the GNP, in general, can be assumed as an arbitrarily oriented ellipsoid. Experimental data in the later part show that this assumption is quite well valid. 
The polarizability tensor of the GNP is measured in a 2-dimensional plane ( $x-z$ plane) as depicted in Fig. 1(b). The projection of an arbitrary ellipsoid onto a 2-dimemsional plane makes an ellipse. The complete 3-dimensional polarizability tensor, the ellipsoid, can be obtained by performing this 2-dimensional measurement in three orthogonal directions and by combining them.

From now on, we describe how the polarizability tensor values are correctly read out from two scattering measurements - RPAE and RPE. In RPAE, the incident laser light of $\lambda=780$ $\mathrm{nm}$ is loosely focused at the tip end using a lens of $10 \mathrm{~cm}$ focal length while rotating the incident polarization using a half wave plate. The scattered light is polarization analyzed by rotating a linear polarizer (analyzer) in front of an APD (C-4777-01, Hamamatsu) in the direction of the incidence. The experimental schematic is shown in Fig. 2(a). For a suitably small GNP, below $100 \mathrm{~nm}$ in diameter, the far-field scattering is dominated by the electric dipole radiation. In that case, the polarizability tensor is described by a $2 \times 2$ matrix in a 2dimensional plane. Here, we assume a simple case of the polarizability tensor $(\vec{\alpha})$ which is symmetric to the laboratory frame with null values of off-diagonal terms, $\vec{\alpha}=\left(\begin{array}{ll}a & 0 \\ 0 & b\end{array}\right)$. A general case of an arbitrarily oriented ellipse with non-zero off-diagonal terms will be discussed in later part. Fig. 2(b) shows the polar plots of the square rooted intensity of the scattered light as a function of the analyzer angle. Three polar plots are shown for the incident polarizations of 0 (black solid), 45 (black dashed), and 90 (black dotted) degrees from the $x$-axis of the laboratory frame. The incident and scattered lights are related through the following relations:

$$
\begin{gathered}
\vec{p}=\vec{\alpha} \vec{E}_{\text {inc }} \\
\vec{E}_{\text {dipole }}=\frac{1}{4 \pi \varepsilon_{0}}\left\{k^{2}(\vec{n} \times \vec{p}) \times \vec{n} \frac{e^{i k r}}{r}\right\}
\end{gathered}
$$

,where $\vec{p}, \vec{E}_{i n c}$, and $\vec{E}_{\text {dipole }}$ are induced dipole moment at GNP, incident electric field, and far-field radiated electric field by the induced dipole moment $\vec{p}$, respectively. Here, $\vec{n}$ is the detection position vector and to be $-\hat{y}$ in the configuration of Fig. 2 . The maxima of the polar plot for every incident polarization (red dots) make an ellipse with the semi-major and the semi-minor axes to be $b$ and $a(b>a)$, respectively. From this, the original tensor value can be extracted.

In the next, RPE is applied in measuring the same polarizability tensor, $\vec{\alpha}=\left(\begin{array}{ll}a & 0 \\ 0 & b\end{array}\right)$. Here, the incident beam polarization is also varied, but with no analyzer in front of the APD as shown in Fig. 2(a). The outer black curve in Fig. 2(c) shows the polar plot of the square rooted scattered light intensity as a function of the incident polarization. When the incident beam polarization is $\theta$, the polarization angle of the scattered light is given as

$$
\varphi=\tan ^{-1}\left(\frac{b}{a} \tan \theta\right)
$$

, and the square rooted intensity is calculated as, 


$$
r_{1}=r_{2}=\left(\frac{a^{2}+b^{2} \tan ^{2} \theta}{1+\tan ^{2} \theta}\right)^{-\frac{1}{2}} .
$$

The inner ellipse and the outer polar plot do not encounter each other except the maxima and the minima of the black curve which correspond to the major and the minor axes of the inner ellipse. By taking the four maxima and minima points of the outer polar plot, the inner ellipse - the polarizability tensor, can be determined. This RPE takes less measurement time compared to the RPAE, but it is less accurate since it has less number of data points. In addition, to apply RPE, it is required a prior study on whether the scatterer is a good dipolar scatterer, otherwise the measured data can be misleading.
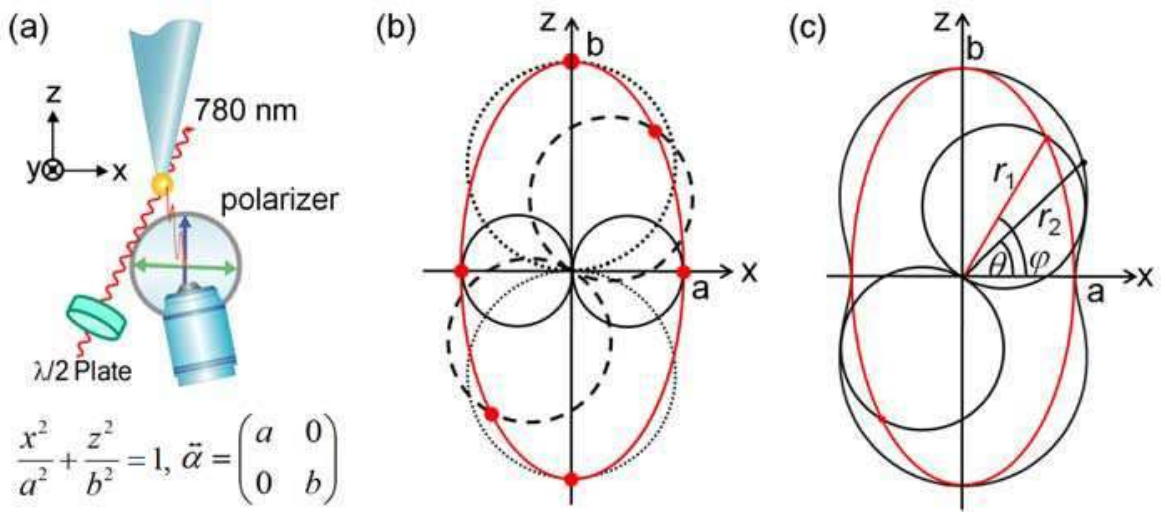

Fig. 2. RPAE vs RPE. (a) Schematics of RPAE (with polarizer) and RPE (without polarizer). A $780 \mathrm{~nm}$ plane wave is focused at the tip end part using a lens of $10 \mathrm{~cm}$ focal length, while rotating the incident polarization using a half wave plate. (b) RPAE. The scattered light is polarization analyzed rotating an analyzer (linear polarizer) in front of the detector. Three black polar plots show the square rooted scattered light intensities as a function of the analyzer angle, for cases of the incident polarization of 0 (solid), 45 (dashed), and 90 (dotted) degrees from the x-axis. The red ellipse is constructed by connecting the maxima of the polar plot of every incident polarization. (c) RPE. APD measures the total intensity of scattered light. The outer black polar plot shows the square rooted scattered light intensity as a function of the incident polarization. The inner red ellipse is determined by choosing the maxima and the minima of the polar plot (black curve) as the semi-major and the semiminor axes of an ellipse (red). From (Lee et al., 2009). (c) 2006 The Korean Physical Society.

So far, we assumed a polarizability tensor of a simplified case - symmetric to the $\mathrm{x}$ and $\mathrm{z}$ axes. But in a general case, the off diagonal elements have non-zero values which implies that the major axis of the ellipse has a deviated angle from the laboratory axes. With $\beta(\geq 1)$ defined as the length ratio between the major and the minor principal axes and $\zeta$ as the angle between the major axis of the scatterer and the laboratory frame $x$-axis, the polarizability tensor can be written as,

$$
\vec{\alpha}=\left(\begin{array}{cc}
\cos \zeta & -\sin \zeta \\
\sin \zeta & \cos \zeta
\end{array}\right)\left(\begin{array}{cc}
\beta & 0 \\
0 & 1
\end{array}\right)\left(\begin{array}{cc}
\cos \zeta & \sin \zeta \\
-\sin \zeta & \cos \zeta
\end{array}\right)=\left(\begin{array}{cc}
\beta \cos ^{2} \zeta+\sin ^{2} \zeta & (\beta-1) \sin \zeta \cos \zeta \\
(\beta-1) \sin \zeta \cos \zeta & \beta \sin ^{2} \zeta+\cos ^{2} \zeta
\end{array}\right) .
$$



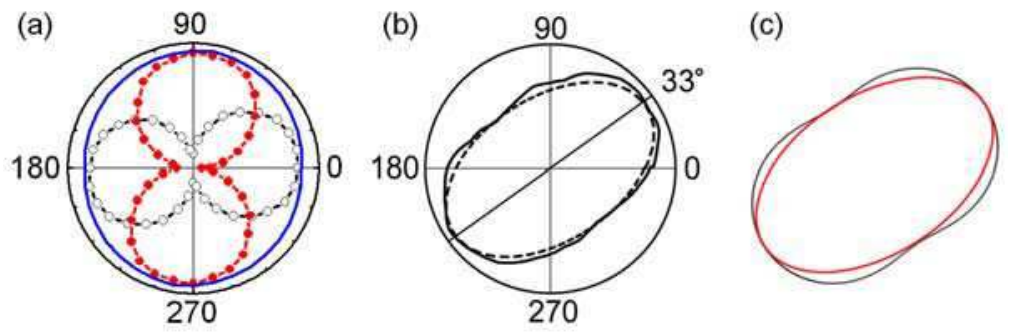

Fig. 3. (a) Two peanut shaped polar plots are measured applying RPAE. The incident polarization angle values are 0 (black open circles) and 90 (red filled circles) degrees. The blue curve is obtained applying RPE. (b) Another measurement applying RPE. Solid curve shows the measured data and the inner dashed ellipse is made by choosing the maxima and the minima of the polar plot as the semi-major and the semi-minor axes. (c) Red ellipse is same as the dashed one in (b), and the outer black curve shows the re-calculated polar plot from the ellipse parameters. From (Lee et al., 2009). (c) 2006 The Korean Physical Society.

Fig. 3 shows experimentally measured data of two GNP attached tips applying RPAE and RPE. Two peanut shaped polar plots in Fig. 3(a) show the square rooted scattered light intensity with the incident polarization directions parallel to the $\mathrm{x}$-axis (black open circles) and the z-axis (red filled circles) of the laboratory frame. The dipolar radiation pattern of the polar plots shows that the attached GNP is a good dipolar scatterer. The outer blue curve is obtained applying RPE. It shows the polar plot of the square rooted scattered light intensity as a function of the incident polarization. From these measurements, values of $\beta$ and $\zeta$ are determined as 1.05 and 80 degrees, respectively, and the resultant polarizability tensor in $\mathrm{x}-\mathrm{z}$ plane is given as $\vec{\alpha}_{1}=\left(\begin{array}{cc}1 & 8 \times 10^{-3} \\ 8 \times 10^{-3} & 1.04\end{array}\right)$. In this case, the GNP shape is quite circular and the off diagonal terms are negligible compared to the diagonal terms. Note that with a small ellipticity ( $\mathrm{a} \approx \mathrm{b}$ in Fig. 2(c)), the polar plot by RPE directly gives the ellipse of the polarizability tensor from Eqs. (3) and (4). The maxima of two polar plots by RPAE clearly touch the polar plot obtained from RPE demonstrating that two methods give a consistent result. Fig. 3(b) shows a scattering measurement of a different GNP functionalized tip obtained by applying RPE. Here, $\beta$ and $\zeta$ values are 1.62 and 33 degrees, and the resulting polarizability tensor is given as $\vec{\alpha}_{2}=\left(\begin{array}{cc}1.208 & 0.257 \\ 0.257 & 1\end{array}\right)$. In this case, the scattering function of the GNP (inner dashed line of Fig. 3(b)) is quite elliptical and the major axis is rotated from the laboratory frame. To check the quality of the measured data, the polar plot is fitted with the determined $\vec{\alpha}$ (black curve in Fig. 3(c)). The similar shape of the polar plots in Figs. 3(b) and 3(c) validates the model and the measurement.

To conclude this section, we demonstrate how the optical response of the gold nanoparticle attached tip, the polarizability tensor, can be measured from two different scattering measurements - RPAE and RPE. RPAE takes more time in determining the tensor values but generally it is more accurate. And also it gives the information of the dipole nature of the tip. In a comparison, RPE takes less time in measuring, but this method needs a prior knowledge whether the tip is a good dipolar scatterer. For our cases, the diameter of GNP attached to the tip is less than $100 \mathrm{~nm}$, the electric dipole radiation is dominant in the far- 
field scattering process and two scattering measurements give a consistent result. The measured data is clearly well reconstructed by a simple calculation validating our analysis methods.

\subsection{Polarization detection of light scattered off GNPs}

A general elliptical polarization state of the local electric field at a fixed position $\vec{r}$ in the $\mathrm{x}-\mathrm{z}$ plane can be written as (Born \& Wolf, 1999):

$$
\vec{E}_{\text {Local }}(\vec{r})=\left(E_{x}, E_{z}\right)=\left(a_{1} e^{i \omega t+i \delta_{1}}, a_{2} e^{i \omega t+i \delta_{2}}\right),\left(a_{1}, a_{2}>0\right),
$$

This field vector rotates at a frequency $\omega$ along the perimeter of an ellipse. A dipole scattering tip gives a scattered far-field $\vec{E}_{S} \propto \vec{\alpha} \cdot \vec{E}_{\text {Local }}$ where $\vec{\alpha}$ is the polarizability tensor of the scatterer (Eqs. (1), (2)). In determining of the polarization states of scattered light, we apply the RAE and the Stokes parameter measurement (Stokes, 1852).

Firstly, the polarization state of an arbitrarily shaped light is determined by the RAE method in which a linear polarizer, mounted inside the optical path of the scattered light and in front of the detector, is rotated by $360^{\circ}$ in $10^{\circ}$ steps. The detected field intensity passing through a polarizer is then given as

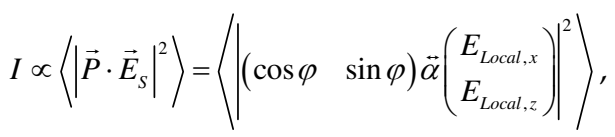

where $\varphi$ is the detecting polarizer angle from the $x$-axis and $\langle\ldots\rangle$ denotes a time average over many optical cycles.

The polar diagram $\sqrt{I(\varphi)}$ shown in Fig. 4(a), recorded by rotating the polarizer in $10^{\circ}$ steps, allows us to determine the polarization state of the scattered light depicted as a red colored ellipse. The major axis angle of the ellipse corresponds to the detecting polarizer angle at which the measured intensity has its maximum and the major and minor axes lengths are proportional to the square-root of the maximum and minimum intensities, respectively. In this way, the shape of the polarization ellipse of the scattered field $\left(\vec{E}_{S}\right)$ is reconstructed. One experimental polar diagram $\sqrt{I(\varphi)}$ is explicitly shown in Fig. 4(b): a gold nano-particle functionalized tip sits at a selected position and scatters a standing wave created by two counter-propagating evanescent waves on a prism surface. The corresponding ellipse is denoted in red color. In case of a highly elliptical polarization as in the standing wave which is our main interest in this section, we denote this ellipse with a double arrowed linear vector (red arrow) for a better visualization. Finally the polarization state of the local field $\vec{E}_{\text {Local }}$ is then reconstructed by a back-transformation $\vec{\alpha}^{-1} \cdot \vec{E}_{S}$ (black arrow), for an example. The missing information is the sense of rotation and the absolute phase, i.e., the point on the ellipse at $t=0$, of the field vector. For a partially polarized light which contains certain amount of un-polarized light, it needs a careful analysis of data to be distinguishable from an elliptical polarization. Therefore, RAE is useful only for highly elliptical polarizations.

The Stokes parameter measurements can be applicable to address the missing information from RAE, such as sense of rotation and degree of polarization - the intensity of the polarized portion to the total intensity. The Stokes parameters are composed of 4 quantities 
(a)

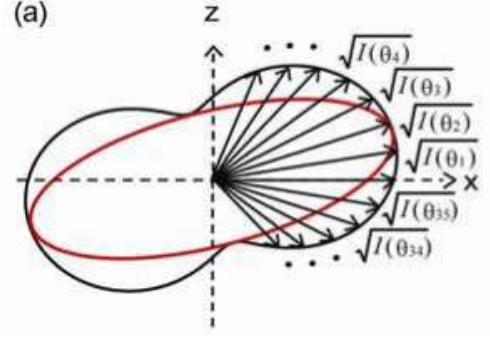

(b)

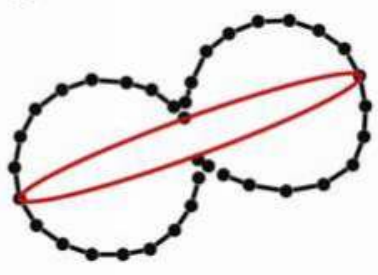

(c)

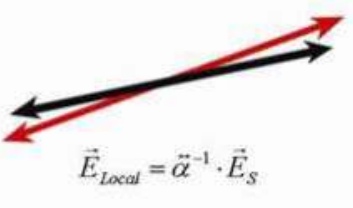

Fig. 4. (a) The outer-plot (black line) results from a polar plot of the squared-rooted intensities for every detecting polarizer angle. The angle $\left(\theta_{\max }\right)$ of the measured intensity maximum corresponds to the major axis angle and the square-rooted maximum (minimum) intensity is proportional to the major (minor) axis length. (b) One such experimental polar plot of the scattered light at one selected position is shown as filled circles. The black line is a guide to the eye. The elliptical polarization state is reconstructed (inner red line). (c) The red arrow represents the long axis of the ellipse shown in (b). By back-transformation using the experimentally determined polarizability tensor of the scatterer, the local field vector is determined (black arrow). From (Lee et al., 2007c). (C) 2007 Optical Society of America.

$\left(s_{0} s_{1} s_{2} s_{3}\right)$ which can be measured by using a combination set of a phase retarder $(\lambda / 4)$ and a linear polarizer (Stokes, 1852; Born \& Wolf, 1999),

$$
\begin{aligned}
& s_{0}=I\left(0^{\circ}, 0\right)+I\left(90^{\circ}, 0\right)=\left\langle a_{1}^{2}\right\rangle+\left\langle a_{2}^{2}\right\rangle, \\
& s_{1}=I\left(0^{\circ}, 0\right)-I\left(90^{\circ}, 0\right)=\left\langle a_{1}^{2}\right\rangle-\left\langle a_{2}^{2}\right\rangle=s_{0} \cos 2 \chi \cos 2 \psi, \\
& s_{2}=I\left(45^{\circ}, 0\right)-I\left(135^{\circ}, 0\right)=\left\langle a_{1} a_{2} \cos \left(\delta_{1}-\delta_{2}\right)\right\rangle=s_{0} \cos 2 \chi \sin 2 \psi, \\
& s_{3}=I\left(45^{\circ}, \frac{\pi}{2}\right)-I\left(135^{\circ}, \frac{\pi}{2}\right)=\left\langle a_{1} a_{2} \sin \left(\delta_{1}-\delta_{2}\right)\right\rangle=s_{0} \sin 2 \chi .
\end{aligned}
$$

Here, $I(\theta, \varepsilon)$ represents the measured light intensity with the linear polarizer angle $\theta$ from the $x$-axis in the laboratory frame, when a phase retardation $\varepsilon$ is given to the z-component relative to the $\mathrm{x}$-component by a $\lambda / 4$ plate. The bracket means the time average over many oscillation periods. $\chi$ and $\psi$ are parameters of the ellipse shown in Fig. 5. The parameter $s_{0}$

\section{(a)}

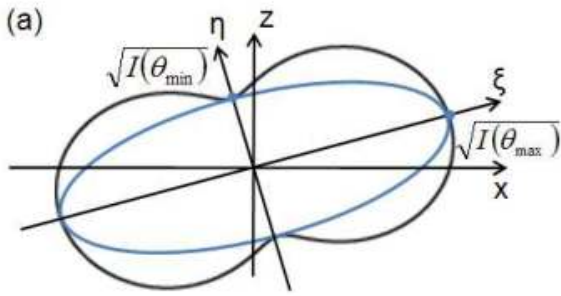

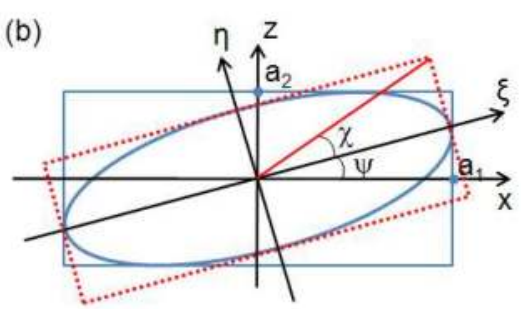

Fig. 5. RAE vs Stokes parameters. (a) The outer-plot (black line) results from a polar plot of the squared-rooted intensities for every detecting polarizer angle. (b) Parameters of an ellipse. Major axis angle $\psi$ defines the orientation of an ellipse. The magnitude and the sign of angle $\chi$ characterize the ellipticity and the rotational sense, respectively. 
represents the total intensity. The parameter $s_{1}$ determines whether the major axis is closer to the horizontal $(x)$ or the vertical $(z)$ axes. In the same analogy, the parameter $s_{2}$ tells whether the major axis is closer to the $x z\left(45^{\circ}\right)$ or $-x z\left(135^{\circ}\right)$ directions. From these three parameters $\left(s_{0} s_{1} s_{2}\right)$, the same amount of information can be derived comparing to the RAE, i. e., the values of $\psi(0 \leq \psi \leq \Pi)$ and $|\chi|(-\Pi / 4 \leq \chi \leq \Pi / 4)$. The final parameter $s_{3}$ represents the intensity difference between the right-handed polarization and the left-handed polarization - the sense of rotation (sign of $\chi$ ). Additionally the Stokes parameters can define the degree of polarizaton, however, in our measurements, we use a monochromatic laser light as a light source and do not discuss this quantity in detail. One missing information of the phase can be determined by applying interferometric methods.

\subsection{Reconstruction of local polarization vectors and tip shape independence}

Due to a relatively simple way of picking up process, fabrication of the GNP funcitonalized tip is reliable and highly reproducible compared to other types nano-probes. Neverthless, the optical properties of a nano sized object are strongly dependent on its shape, size, and orientation. For an example, the polarization state of the scattered light is strongly dependent on the scattering function of this dipole scatterer, i.e., its polarizability tensor, it is important to characterize each tip carefully before the local electric field orientation is reconstructed.

To investigate how the effect of different tips can be corrected in the final determination of the local polarization vector, we prepared three tips attached with gold nanoparticles of different shapes and sizes. The corresponding polarizability tensor of each of these tips is measured as described in the section of 2.1. Using these tips we measured the polarization state of a standing wave generated on a prism surface. Our experimental setup is schematically depicted in Fig. 6(a). A p-polarized plane wave is guided into a prism and generates, with its reflected wave from the mirror at the other side of the prism, an evanescent standing wave on the prism surface, if the incident angle $\theta_{i}$ is set to be larger than the total internal reflection angle $\theta_{c}=\sin ^{-1}\left(n_{\text {air }} / n_{\text {prism }}\right)$ given by the refractive indices of two media. For an evanescent standing wave, generated by two counter-propagating ppolarized beams of equal intensity, the field vector is given by:

$$
\vec{E}(\vec{r})=\left(E_{x}, 0, E_{z}\right)=E_{0}\left(\cos k x, 0,-\frac{k}{\kappa} \sin k x\right) e^{-\kappa z}
$$

where $E_{0}$ is a constant magnitude. $k$ and $\kappa$ are related by the Helmholtz equation: $k^{2}-\kappa^{2}=\left(\frac{\omega}{c}\right)^{2}$ and are determined by the angle of incidence and the index of refraction of the prism. In Fig. 6(b), theoretically calculated horizontal and vertical field intensities, $\left|E_{x}\right|^{2}$ and $\left|E_{z}\right|^{2}$, respectively, of this evanescent standing wave are presented with the corresponding field vectors of polarization shown in the upper part. For an incident angle of $\theta_{i}=60^{\circ}$ and $n_{\text {prism }}=1.51$ at $\lambda=780 \mathrm{~nm}$, the peak vertical field intensity is about 2.25 times larger than its horizontal counterpart, and these two field components are spatially displaced with a $90^{\circ}$ shift in their intensity profiles.

We scanned the prism surface along the $\mathrm{x}$-direction and the polarization characteristics of photons scattered by these GNP attached tips are analyzed applying RAE method. The tip to sample distance was controlled to be constant using a shear force mode feedback system 

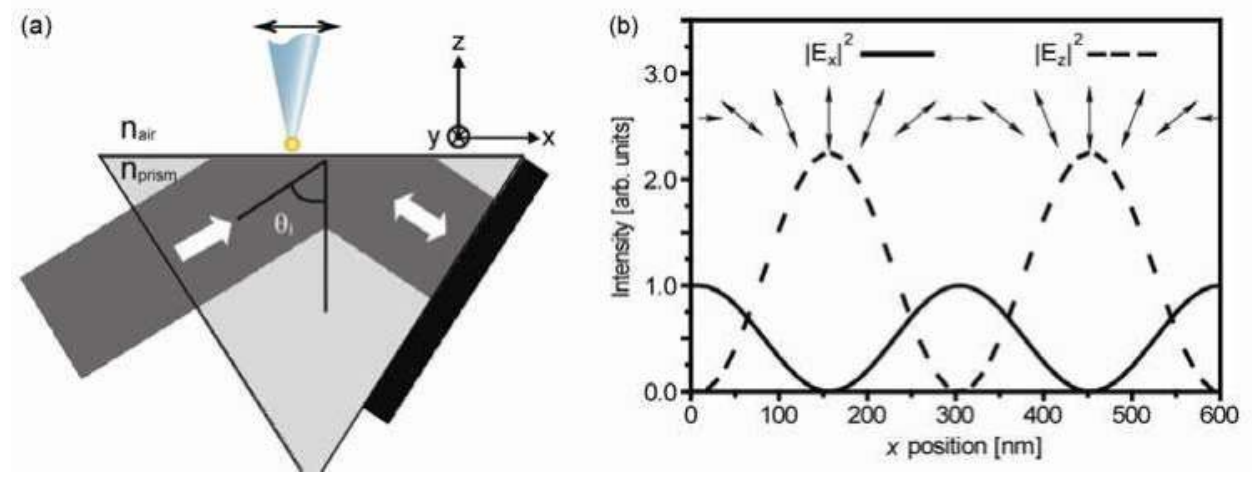

Fig. 6. (a) Experimental setup: A $780 \mathrm{~nm} \mathrm{cw}$-mode Ti:Sapphire laser enters at normal incidence into one side facet of an equilaterally shaped prism and is retro-reflected at the other side facet to generate an evanescent standing wave on the top surface. The gold nanoparticle attached tip scatters the local fields into far-field region. (b) Theoretically calculated local field components as a function of the scatterer position: vertical $\left|E_{z}\right|^{2}$ (dashed line) and horizontal $\left|E_{x}\right|^{2}$ (solid line) component, respectively. The corresponding local field vectors of polarization are presented at every position.

and the detection angle was set about $20^{\circ}$ from the prism surface (-y axis) due to the experimental restrictions. The effects of the detection angle from the surface on the image contrast will be discussed later.

Fig. 7 shows the local field vectors of polarization obtained within a scan range of $600 \mathrm{~nm}$ on the prism surface obtained by using three different tips. The corresponding polarizability tensors are indicated above the vector plots. The results for Tip 1 and 2 are obtained with attached gold particles with a diameter of $200 \mathrm{~nm}$ and $100 \mathrm{~nm}$, respectively. In these cases the effective polarizability tensors are close to the identity matrix, which means circular shape of GNPs. The bottom one is obtained with the tip introduced in Fig. 3(b). For all three tips attached with gold nanoparticles of different size and shape, the measured local polarization vectors show a good agreement with the theoretical prediction in Fig. 6(b), demonstrating the independence of the finally determined local polarization vector on the tip shape.

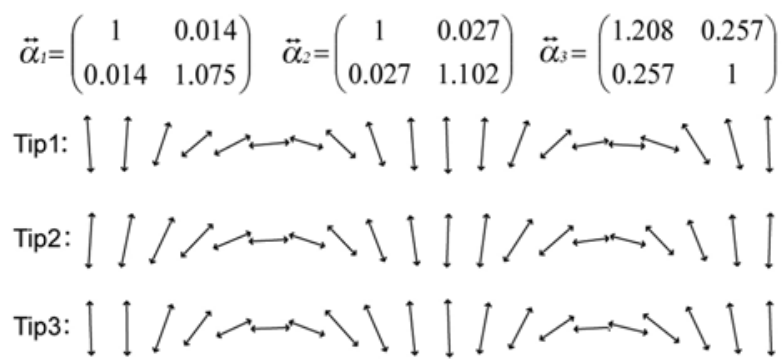

Fig. 7. Local field polarization vectors of the evanescent standing wave generated on the prism surface within a $600 \mathrm{~nm}$ scan range obtained by using three different gold-particle functionalized tips. The corresponding polarizability tensors are displayed above the scans. From (Lee et al., 2007c). (C) 2007 Optical Society of America. 
Finally, Fig. 8 displays the theoretical and experimental vector field maps within a $600 \mathrm{~nm} \times$ $300 \mathrm{~nm}$ scan area in $\mathrm{x}-\mathrm{z}$ plane. The field vector rotates as we move along the $\mathrm{x}$-direction and the electric lines of force are explicitly visualized. The reconstructed field polarization vectors match well with those expected for the evanescent surfaces waves unperturbed by the tip. Generally one may expect a certain perturbation of the local electric field by the field scatterer. The demonstrated ability to quantitatively map electric field vectors of local polarization in simple cases, such as the standing surface waves investigated here, will certainly be useful in obtaining a deeper understanding of the interaction between the tip scatterer and localized electric fields at surfaces. In the section of 3 , the surface effects on the far-field detected light scattered from the near-field region will be discussed in more details.
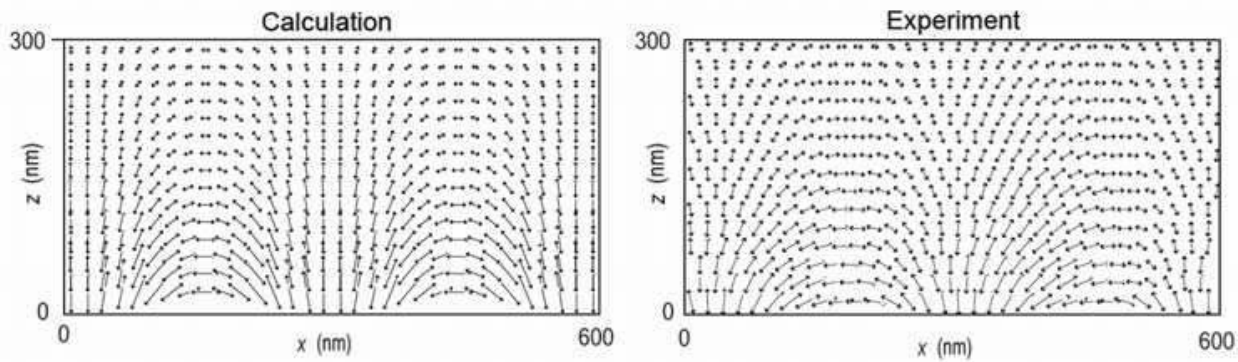

Fig. 8. Vector field plot of an $\mathrm{x}-\mathrm{z}$ area of $600 \mathrm{~nm}$ by $300 \mathrm{~nm}$ of the theoretical (left) and experimental (right) results, respectively. From (Lee et al., 2007a). (c) 2007 Nature Publishing Group.

Before ending up this section, we need to check the validness of dipole approximation of GNPs when the measurements are carried out in the evanescent near-fields. With higher values of $k$-vector, the evanescent field is confined to the sample surface and exponentially decays to the direction normal to the surface. The evanescent field generated on the prism surface (BK7), with the incident angle of $\theta_{i}=60^{\circ}$ as depicted in Fig 6, the decay constant in intensity is calculated as $147 \mathrm{~nm}$. Then the far-field scattered field by a GNP of radius $r$ is calculated in the Mie-scattering formalism (Chew, et al., 1979; Ganic et al., 2003).

$$
E_{s c}(r)=\sum_{l=1}^{\infty} \sum_{m=-l}^{l}\left\{\frac{i c_{0}}{n_{1}^{2} \omega} \beta_{E}(l, m) \nabla \times\left[h_{l}\left(k_{1} r\right) X_{l m}\left(e_{r}\right)\right]+\beta_{M}(l, m) h_{l}\left(k_{1} r\right) X_{l m}\left(e_{r}\right)\right\}
$$

Here, we do not include the effect of the glass tip shaft. The relative magnitude of the Miecoefficient of electric and magnetic components for each radius is listed in Table 1. Upto GNP radius value of $100 \mathrm{~nm}$, the electric dipole term dominates. For the case of $r=150$, the magnetic and higher order terms significantly effect on the scattering signal and the dipole approximation cannot be applied anymore.

\begin{tabular}{|c|c|c|c|}
\hline & $r=50 \mathrm{~nm}$ & $r=100 \mathrm{~nm}$ & $r=150 \mathrm{~nm}$ \\
\hline$\left|a_{1}\right|$ & 1 & 1 & 1 \\
\hline$\left|a_{2}\right|$ & 0.059 & 0.026 & 0.014 \\
\hline$\left|b_{1}\right|$ & 0.065 & 0.124 & 0.250 \\
\hline$\left|b_{1}\right|$ & 0.003 & 0.003 & 0.021 \\
\hline
\end{tabular}

Table 1. Magnitude of two lowest orders of the Mie-coefficients $a_{l}$ (electric) and $b_{l}$ (magnetic). 


\subsection{Three dimensional expansion of local field polarization vector detection}

Expanding the local polarization vector detection into a full 3-dimensional space, in principle, is straight forward by combining of 2-dimensional measurements in two orthogonal directions. As a target field, we chose a focused radial polarized light. The intense longitudinal field at the focus center of a radially polarized beam has attracted many attentions not only in a theoretical point of view but also in application respects such as confocal microscopy, optical data storage, and particle trapping and acceleration of particles. Generating good quality cylindrical vector beams, radially and azimuthally polarized beams, has been an intense research area itself. Several different methods are presented interferometry, twisted liquid crystal, and laser mode controlling inside the cavity.

The interesting axis symmetric field distribution of the cylindrical beam at the focus stems from its axis symmetry of the field polarizations. The field configuration of the cylindrical beam has been demonstrated in theoretical works (Youngwoth \& Brown, 2000), but it has been challenging to fully demonstrate it in experiment.

Experimental demonstration starts with a 3-dimensional tip characterization. Here, we adapted a slightly diffrent method to reduce down the total measuring time. Tip end is illuminated by loosely focused Ti:Sapphire laser beams in three orthogonal directions with various incident beam polarizations (Fig. 9(a)). The scattered electric field $\left(\vec{E}_{s c a}\right)$ is detected in $\left(\begin{array}{lll}1 & \pm 1 & 0\end{array}\right),\left(\begin{array}{lll}1 & 0 & 0\end{array}\right)$ and $\left(\begin{array}{lll}0 & -1 & 0\end{array}\right)$ directions for each incident beam direction. With assuming the attached GNP as a dipolar scattering center, the incident and the scattered electric fields are related through the polarizability tensor $\vec{\alpha}$ (Ellis \& Dogariu, 2005):

$$
\vec{E}_{s c a}=\vec{\alpha} \cdot \vec{E}_{i n c}=\sum_{i, j=1}^{3} \alpha_{i j} E_{i n c, j} .
$$

(a)

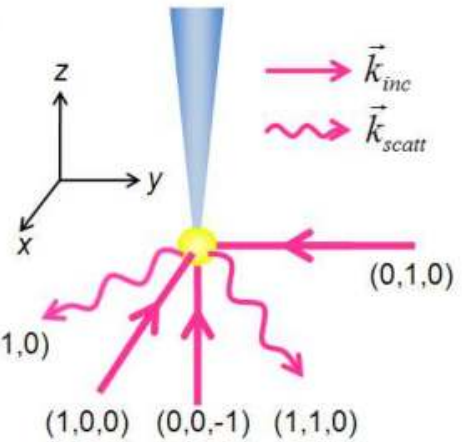

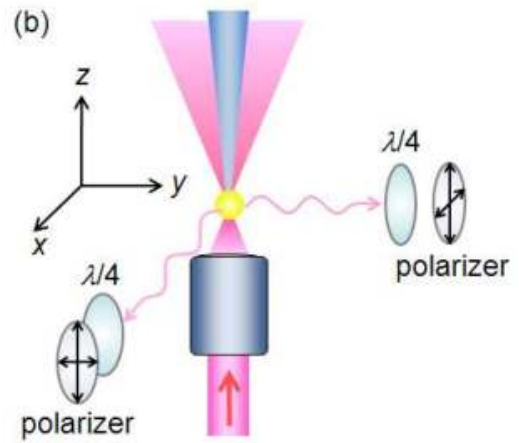

Fig. 9. (a) Three dimensional tip characterization. The tip end is illuminated by Ti:Sapphire laser beams in three orthogonal directions in sequence varying the incident beam polarization. The scattered electric field is detected in the direction of incidence and also in $(1 \pm 10)$. (b) Polarization vector mapping of a focused radially polarized light. A radially polarized beam generated by using a radial polarization converter is focused by an objective. A GNP functionalized tip is scanned the focusing area in three dimensional space using a 3-axes nano positioner (Nano Cube, Physik Instrumente). The polarizaton state of the scattered light is determined by applying the RAE and measuring the Stokes parameters in two orthogonal axes. 
From the pre-adjusted incident electric field and the measured scattered electric field polarization states, the polarizability tensor values are directly calculated from Eq. (11):

$$
\vec{\alpha}=\left(\begin{array}{ccc}
1.01 & 0.18 & -0.21 \\
0.18 & 0.70 & 0.12 \\
-0.21 & 0.12 & 1
\end{array}\right) .
$$

The radial polarization can be described as combination of Hermite-Gaussian modes:

$$
\text { Radial polarization }=H G_{10} \hat{x}+H G_{01} \hat{y}
$$

The electric field at the focus in the Cartesian coordinate is given as follows (in air) (Youngworth \& Brown, 2000; Novotny \& Hecht, 2006):

$$
E(\rho, \varphi, z)=\left(\begin{array}{l}
E_{x} \\
E_{y} \\
E_{z}
\end{array}\right)=\frac{i k f^{2}}{2 w_{0}} E_{0} e^{-i k f}\left(\begin{array}{c}
i\left(I_{11}-I_{12}\right) \cos \varphi \\
i\left(I_{11}-I_{12}\right) \sin \varphi \\
-4 I_{10}
\end{array}\right),
$$

where

$$
\begin{aligned}
& I_{10}=\int_{0}^{\theta \max } f_{w}(\theta) \sqrt{\cos \theta} \sin ^{3} \theta J_{0}(k \rho \sin \theta) e^{i k z \cos \theta} d \theta \\
& I_{11}=\int_{0}^{\theta \max } f_{w}(\theta) \sqrt{\cos \theta} \sin ^{2} \theta(1+3 \cos \theta) J_{1}(k \rho \sin \theta) e^{i k z \cos \theta} d \theta \\
& I_{12}=\int_{0}^{\theta \max } f_{w}(\theta) \sqrt{\cos \theta} \sin ^{2} \theta(1-\cos \theta) J_{1}(k \rho \sin \theta) e^{i k z \cos \theta} d \theta \\
& f_{w}(\theta)=\exp \left(-f^{2} \sin ^{2} \theta / w_{0}^{2}\right) .
\end{aligned}
$$

Here, $J_{\mathrm{n}}$ is the $n$ th-order Bessel function and $k$ is the wave vector of the incident beam. The focal length $f$, maximum focusing angle $\theta_{\max }$, and the incident beam radius $w_{0}$ are related as follows: $\frac{w_{0}}{f}=\sin \theta_{\max }=N A$ (effective numerical aperture of the objective).

A radially polarized light is generated by using a radial polarization converter (Arcoptix) and focused by an objective (NA=0.39). A GNP functionalized tip scans the focus area and the scattered light is polarization analyzed in two orthogonal directions by applying the RAE and by measuring the Stokes parameters. The local polarization state of the focused light is reconstructed by performing the back transformation of the polarizability $\vec{\alpha}$ obtained above in Eq. (12) to the scattered electric field.

Fig. 10 shows the local electric field components in the focus plane $(z=0)$. Upper three intensity plots show the experimentally measured electric field components. As predicted by calculations as shown below, vertical field intensity is a maximum at the center of the focus. On the other hand, the $x$ - and the $y$-components have intensity minima at the same spatial position. Combined image of (b) and (c) generates a donut shaped intensity distribution for the transversal field component (not shown). The NA value of the used oil immersion objective $\left(n_{\mathrm{oil}}=1.50 \sim 1.51\right)$ is 1.45 with full using the back aperture. The effective 
NA value for this measurement performed in air side is chosen as 0.39 from the incident beam waist $\left(w_{0}=2 \mathrm{~mm}\right)$ and the back aperture radius of the objective $(5 \mathrm{~mm})$. Experiment and calculation agree well each other in the focused beam size and also in the relative intensity peak ratio between the transversal and the vertical components.
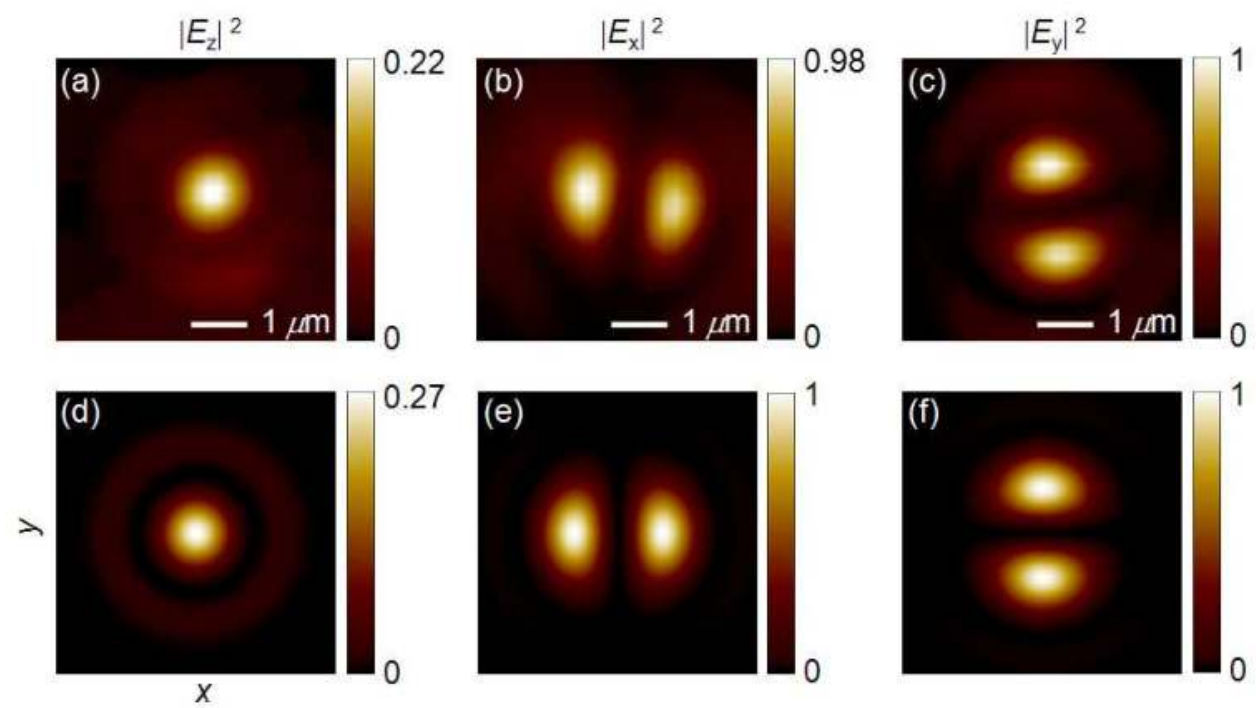

Fig. 10. (a-c) Experimentally measured field intensity distribution profiles for three orthogonal axes. (d-f) Numerically calculated field intensity distribution of the corresponding field component in the focus plane. From (Ahn et al., 2009). (c) 2009 Optical Society of America.

The 3-dimensional polarization vectors are shown in Fig. 11 determined from the RAE (a) and the Stokes parameters (b). They show quite complicated features, and the top and the side views of (b) are shown below in (c) and (d), respectively. In the top view (c), the polarization direction, the long axis of the ellipse, directs to the focus center. It tells that the transversal component still has a radial polarization state at the focus. However, due to a slight deviation of the beam axis from the z-axis, there are elliptical polarization states in the transversal field components unlike the calculations (Youngworth \& Brown, 2000; Novotny $\&$ Hecht, 2006). Fig. 11(d) shows the side view $(y=0)$ of $(b)$ for several different tip height $(z)$ values. Note that the vertical field amplitude is magnified by 5 times in this figure for a better visualization. The optical axis of the focused beam is slightly deviated from $-x$ to $x$ direction as it propagates from $-\mathrm{z}$ to $\mathrm{z}$ direction. It directly shows the imperfectness of the beam alignment together with the details of the focused radially polarized light.

In this section, a full 3-dimensional local polarization vector detection is demonstrated. This is achieved by performing the 2-dimensional polarization vector detection in two orthogonal directions and by combining them. Focused radially polarized light is a good target field due to an intense longitudinal field component at the focus center. The $3 \times 3$ polarizability tensor values of the GNP functionalized tip are also obtained by performing the scattering measurement in three orthogonal axes. The polarization vectors of a focused radially polarized light are mapped applying the RAE and the Stokes measurement. 

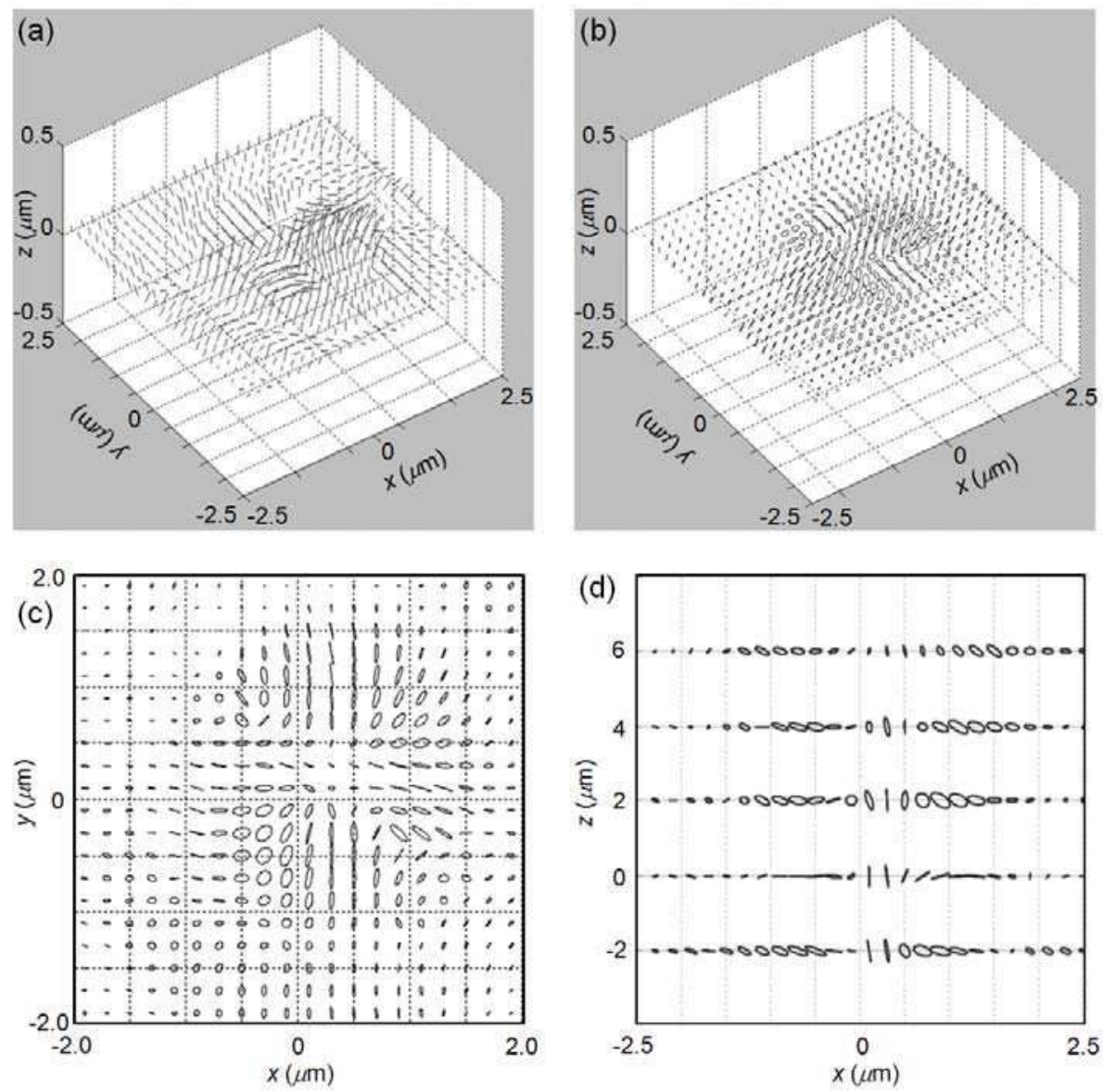

Fig. 11. Polarization vector mapping in the focus plane $(z=0)$ by the (a) RAE and the (b) Stokes measurement. (c) Top view of $(b)$. (d) Side view $(y=0)$ of $(b)$ for several tip height $(z)$ values. The vertical field amplitude $\left(E_{\mathrm{z}}\right)$ is 5 times multiplied in $(\mathrm{d})$ for a better visualization. From (Ahn et al., 2009). (C) 2009 Optical Society of America.

\section{Sample surface effects on local field detection in near field region: image dipole effects}

Unlike the light scattering by a tip in a homogeneous media, the scattered light in a near field region suffers significant modifications due to the existence of the surface. In the apertureless near-field scanning optical microscopy, the dipolar coupling between the real dipole at the tip apex and its image dipole induced at the sample surface has been widely applied in the analysis of the far-field scattered signals (Knoll \& Keilmann, 2000; Raschke \& Lieanu, 2003; Cvitkovic et al., 2007).

In this section, we systematically investigate the polarization dependent image dipole effects on the near-field polarization vector detection on a dielectric and a flat metal ( $\mathrm{Au}$ ) surfaces. 
The experimental schematic is depicted in Fig. 12(a). A $780 \mathrm{~nm} \mathrm{cw}$ Ti-sapphire laser is guided on one facet of the equilateral shaped prism (BK7) to generate a propagating evanescent wave at the air-prism interface. With the incident angle $\theta_{i}=60^{\circ}$ and the refractive index $n_{\text {prism }}=1.51$ at the wavelength of $780 \mathrm{~nm}$, the intensity ratio of $\left|I_{z}\right| /\left|I_{x}\right|$ and the skin depth into air are given as 2.25 and $147 \mathrm{~nm}$, respectively, from Eq. (9). The evanescent field is then scattered by a GNP of radius $50 \mathrm{~nm}$ attached on a chemically etched optical fiber tip in a constant height mode $(h \sim 55 \mathrm{~nm})$. Tip was fixed at one selected x-position and the scattered light intensity is polarization analyzed. The relative intensity ratio of the vertical and the horizontal components of the local field at the GNP position is measured while varying the collection angle $\phi$.

To account for the surface effects on the signal, we firstly consider the interference between the direct radiation from the GNP and its reflection from the sample surface to the detector by using a simple image dipole model ((i) in Fig. 12(b)). The reflected light from the surface can be considered as the radiation from the image dipole located at the opposite side to the interface. The relative strength of the radiation from the real and the image dipole is determined by the magnitude of the reflection coefficient $\left(R^{s(p)}=\left|R^{s(p)}\right| e^{i \varphi_{d e l a y s} s(p)}\right)$ of the $s-$ and the $p$-polarized light at air-prism interface. The relative phase difference between the real and the image dipoles is determined by the argument of the reflection coefficient, $\varphi_{\text {delay, }(s, p) \text {, }}$

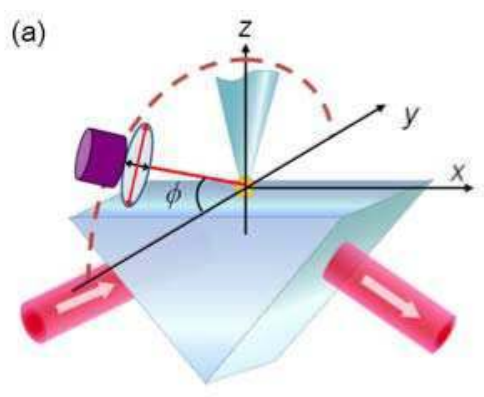

(c)

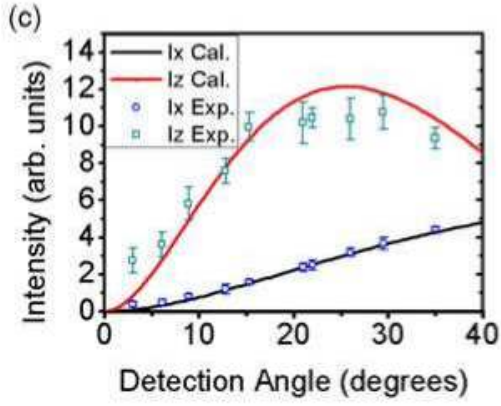

(b)

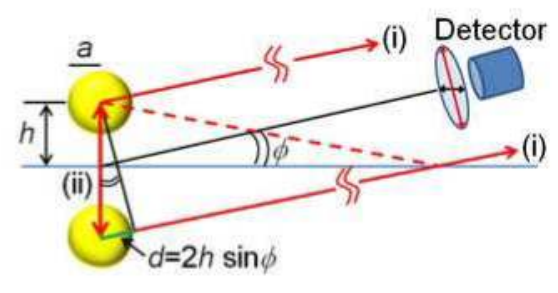

(d)

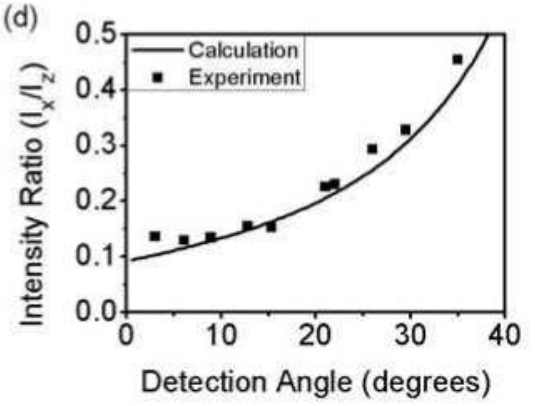

Fig. 12. Image dipole effects on a dielectric surface. (a) Experimental schematics. (b) The reflected light at the sample surface (dashed line) can be considered as the radiated field from the image dipole (i). The mutual interaction of the real (upper) and the image (below) dipoles modifies the radiation properties of their own (ii). (c-d) Relative intensities of the horizontal and the vertical field components of the progating evanescent wave on a prism surface. (c-d) from (Ahn et al., 2008). (C) 2008 Elsevier B.V. 
as well as the phase difference caused by the optical path length difference $\varphi_{\text {diff }}=k_{0} \cdot d$, where $d=2 h \sin \varphi$ is the path difference in Fig. 12(b). For analytical calculations, we applied the single dipole model (SDM) where the real (above the surface) and the image (below) dipoles are assumed to be point-like dipoles. We note that the reflection coefficient of the plane wave is used in this analysis because the scattered light is detected in far-field region. The effects of the higher nonlinear terms included in the effective polarizability change will be discussed in later part.

The signal intensity of the horizontal $(s)$ and the vertical $(p)$ dipoles in the SDM is written as

$$
I_{s(p)}=\left|E_{s(p) \text {-orig }}+E_{s(p)-\text { imag }}\right|^{2}=\left|E_{s(p) \text {-orig }}\right|^{2}\left(1+\left|R_{s(p)}\right|^{2}+2\left|R_{s(p)}\right| \cos \left(\varphi_{\text {delay }, s(p)}+\varphi_{\text {diff }}\right)\right) .
$$

Here, $\left|E_{s(p)-\text { orig }}\right|^{2}$ is determined by the relative time-integrated strength of the horizontal (s) and the vertical $(p)$ field components of the propagating evanescent wave. For the vertical polarization case, i.e., $p$-polarization case, $\cos ^{2} \phi$ term should be multiplied to Eq. (15) to compare with experimentally measured one because of that an oscillating dipole cannot radiate light in its oscillation direction. In a detailed explanation, the electric field at a detection position $\vec{r}$ radiated by a dipole moment $\vec{p}$ located at origin is described as follows (Jackson, 1998);

$$
\vec{E}_{\text {dipole }}=\frac{1}{4 \pi \varepsilon_{0}}\left\{k^{2}(\hat{d} \times \vec{p}) \times \hat{d} \frac{e^{i k r}}{r}+[3 \hat{d}(\hat{d} \cdot \vec{p})-\vec{p}]\left(\frac{1}{r^{3}}-\frac{i k}{r^{2}}\right) e^{i k r}\right\},
$$

where $k$ is the wave-vector and $r$ is the distance from the dipole to the detector. With $\vec{p}=\left(0,0, p_{z}\right)$ and the detection position vector $\hat{d}=(0,-\cos \phi, \sin \phi)$, the radiated electric field in far-field region with consideration of the surface reflection is given by substituting Eq. (16) into Eq. (15).

$$
\vec{E}_{\text {det ector }}=\frac{k^{2} p_{z}\left(1+\left|R_{s(p)}\right|^{2}+2\left|R_{s(p)}\right| \cos \left(\varphi_{\text {delay }, s(p)}+\varphi_{\text {diff }}\right)\right)}{4 \pi \varepsilon_{0} r^{2}}\left(0,-\cos \phi \sin \varphi, \cos ^{2} \phi\right)
$$

Note that the vertical polarizer direction in Fig. 12(a) is differ from the z-axis in the laboratory frame but parallel to $\vec{E}_{\text {detector }}$ giving the measured intensity of the vertical component proportional to $\cos ^{2} \phi$ instead of $\cos ^{4} \phi$.

The relative intensities of the horizontal and the vertical field compoentns as a fuction of the detection angle are shown in Fig. 12(c-d). Simple analytical calculation well predicts the experiemtal result.
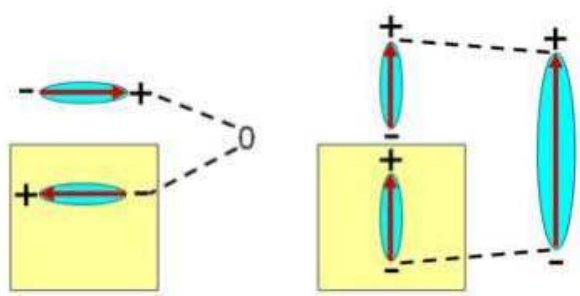

Fig. 13. Polarization direction dependence of the image dipole effects at the vicinity of metal surfaces. 
Image dipole effects are more dramatic in metallic surface. Image dipole effects are highly dependent on the polarization direction, constructive (destructive) interference between real and image dipoles for the vertically (horizontally) aligned one in the vicinity of metal surfaces, respectively.

We use a propagating surface plasmon polaritons (SPP) as an excitation source. The wellcharacterized field profile of SPP and sufficiently reduced background noise by virtue of the evanescent nature of SPP make it possible to carry out quantitative studies of the image dipole effects on metallic surfaces. Fig. 14 shows our experimental schematics. A propagating SPP is generated at the slit position by impinging a beam of cw-mode TiSapphire laser (wavelength $\lambda_{0}=780 \mathrm{~nm}$ ) at the back side of the sample. The incident polarization is adjusted perpendicular to the silt direction for the coupling of the incident light to the SPP. The thickness of the gold film and the slit width are chosen as $80 \mathrm{~nm}$ and $400 \mathrm{~nm}$, respectively, to maximize the SPP coupling efficiency from the incident light (Kihm et al., 2008). A lens (focal length of $5 \mathrm{~cm}$ ) focuses the excitation beam at the slit position to eliminate the position dependent interference between the directly transmitted light through the thin metal film and the propagating SPP at the tip position. The tip is fixed at one selected $x$-position at about $50 \mu \mathrm{m}$ away from the slit exit to diminish unwanted backgrounds resulting from the deflected light at the tip shaft when the propagating light transmitted at the slit position touches the tip surface (Lee et al., 2007b).

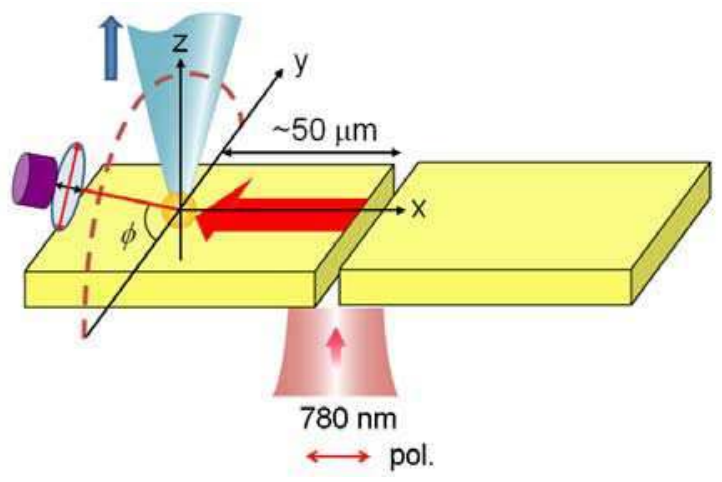

Fig. 14. Schematic diagram of the experimental setup. Tip is placed above a flat gold surface about $50 \mu \mathrm{m}$ away from the slit position. A $780 \mathrm{~nm} \mathrm{cw}$ Ti-Sapphire laser beam is incident from the bottom side of the sample to generate SPPs propagating in $\pm x$-direction on air-gold interface. This propagating SPP is scattered by the GNP functionalized tip and a linear analyzer is placed in front of the detector for the axis resolved detection. The tip-sample distance $(h)$ is varied from near- to far-field region, and the detection angle $(\phi)$ between the sample surface and the detector position vector is also changed.

The electric field of the propagating SPP on a flat gold surface can be described as follows (Reather, 1988);

$$
\vec{E}_{S P P}(\vec{r})=\left(E_{S P P, x}, 0, E_{S P P, z}\right)=E_{0}\left(\cos \left(k_{S P P} x-\omega t\right), 0,-\frac{k_{S P P}}{\kappa} \sin \left(k_{S P P} x-\omega t\right)\right) e^{-\kappa z}
$$


where $E_{0}$ is a constant amplitude, $\kappa=\operatorname{Im}\left[\frac{2 \pi}{\lambda_{0}} \sqrt{\frac{\varepsilon_{a i r}^{2}}{\varepsilon_{\text {air }}+\varepsilon_{A u}}}\right]$ the reciprocal skin depth of the SPP into air $\left(n_{\text {air }}=1\right)$, and $k_{S P P}=\frac{2 \pi}{\lambda_{0}} \sqrt{\frac{\varepsilon_{a i r} \cdot \varepsilon_{A u}}{\varepsilon_{a i r}+\varepsilon_{A u}}}$ the wave-number of the SPP. As a dielectric constant of GNP, $\varepsilon_{\mathrm{Au}} \approx-22.5+1.4 i$ of bulk gold at the wavelength $\lambda_{0}=780 \mathrm{~nm}$ is used (Reather, 1988). The time integrated intensity ratio of the horizontal and the vertical field components of propagating SPP is determined by the dielectric constant of gold.

$$
\frac{I_{S P P, z}}{I_{S P P, x}}=\frac{\left|E_{S P P, z}\right|^{2}}{\left|E_{S P P, x}\right|^{2}}=\left|\varepsilon_{A u}\right|
$$

This propagating SPP induces the dipole moment at the GNP attached to the apex of an etched glass fiber. The scattered light intensity is measured while varying the tip-sample distance $h$ and the detection angle $\phi$ between the sample surface and the detector position vector $\hat{d}=(0,-\cos \phi, \sin \phi)$. A long working objective lens (Mitutoyo M Plan Apo 10×) collects the scattered light and delivers it to an APD. A linear polarizer placed before the detector resolves the polarization direction of the scattered light.

Figure 15(a) shows the plot of the signal intensities versus the tip-sample distance $(h)$ obtained with the detection polarizer oriented along the horizontal (black open circles) and vertical (red open circles) directions to the sample surface. The elliptical scattering shape of the GNP is taken into account by dividing the horizontal signal intensity with $(1.34)^{2}$. Here, the detection angle $(\phi)$ is $33^{\circ}$.

The signal intensity of the horizontal (s) and the vertical $(p)$ dipoles in the SDM is given by Eq. (15). In this case, $\left|E_{s(p)-\text { orig }}\right|^{2}$ is originally determined by the relative time-integrated strength of the horizontal (s) and the vertical (p) field components of the propagating SPP as in Eq. (19). In addition to that, it is also considered the radiating property modifications of the GNP itself $\left(\left|E_{s(p)-o r i g}\right|^{2}\right)$, caused by the reflected fields directly back to the GNP ((ii) in Fig. 12(b)). The radiated field from the real dipole (upper sphere in the Fig. 12(b)) influences the image dipole (below), and the resultant altered field of the image dipole modifies the real dipole again. This mutually repeating effect on the $\alpha_{\text {eff }}$ can be calculated in a self-consistent manner. In previous studies (Knoll \& Keilmann, 2000; Raschke \& Lieanu, 2003), the $\alpha_{\text {eff }}$ is calculated in the quasi-electrostatic limit assuming a small distance from the particle (or the tip apex of a metal tip) to the interface. The tip apex was considered as a point-like scattering center. In this study, in calculating $\alpha_{\text {eff, }}$ all terms of Eq. (16) are included for the case of a wider separation between the tip and the sample surface. Using the SDM and $\alpha_{\text {eff }}$ derived from it, we fail to reproduce the experimentally measured signals (dashed lines in Fig. 15(a)). The relative signal intensities of the vertical and the horizontal components are different in calculation and experiment. In addition, the lifted valley of the vertical polarization signal appeared in experiment at $h \sim 300 \mathrm{~nm}$ of the tip-sample distance cannot be recovered because the magnitude of the reflection coefficient $\left|R_{s(p)}\right|$ is close to unity for all polarization directions and detection angles at the gold-air interface (see Eq. 15). 
In order to understand the origin of the observed deviation, we applied the coupled dipole method (CDM) (Martin et al., 1995; Novotny \& Hecht, 2006) where the GNP of radius $50 \mathrm{~nm}$ is divided into approximately 500 identical sub-volumes, which act as point dipoles. In this calculation, all mutual interactions between sub-volumes including the reflected field from the sample surface are considered. In Fig. 15(a), the theoretical calculations with CDM (solid lines) are compared to the experimental data (open circles) for two orthogonal detection polarizer angle directions. The theory and the experiment are in excellent agreements to each other. Furthermore, the lifted non-zero value of the minimum at $\mathrm{h} \sim 300 \mathrm{~nm}$ is clearly recovered by the theory.

The oscillation period $\frac{\lambda_{0}}{2 \sin \phi}$ is determined by the condition of $\varphi_{\text {diff }}=k_{0} \cdot d=2 \pi$ (Fig. 12(b)). In

Fig. 15(b), the calculated values of oscillation period are plotted in solid line and experimentally measured values for three different detection angles are marked with open circles. For larger detection angles of $\phi=21^{\circ}$ and $33^{\circ}$ the values from the calculations and the experiments agree well to each other, but for $\phi=8.5^{\circ}$ there is a relatively large discrepancy between them. This difference seems to result from the gradually confined numerical aperture (NA) of the collection objective. The used objective lens of NA=0.28 has the collection solid angle $\varphi_{s}=16.3^{\circ}$ (inset in Fig. 15(b)), which means that for a smaller detection angle $\phi<\varphi_{s}$, the lower part of the lens does not collect the signal, implying the bigger value of effective collection angle.

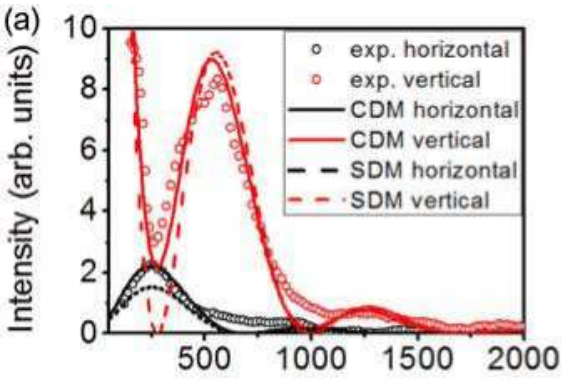

Tip-sample distance $h(\mathrm{~nm})$

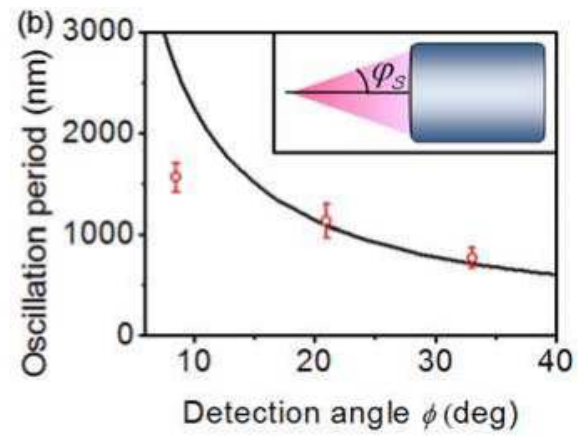

Fig. 15. (a) Experimentally measured tip-sample distance dependent signal intensity with the detection analyzer direction vertical (red) and horizontal (black) to the sample surface. Signal intensities calculated by applying SDM (dashed lines) and CDM in Green-function formalism (solid lines) are shown together with the experimental results (open circles). Here, $\phi=33^{\circ}$. (b) Oscillation periods from the calculation (solid line) and the experiment (open circles). Inset: the solid angle of the objective, $\varphi_{s}=16.3^{\circ}$. From (Lee et al., 2008). (c) 2008 Optical Society of America.

In conclusions, we experimentally demonstrate how the image dipole modifies the far-field detected signal depending on its polarization direction to the dielectric and the metal surfaces. By using propagating evanescent optical wave and SPP as excitation sources, well characterized local dipoles are generated at the GNP. Contributions of dipoles aligned vertically and horizontally to the surface are completely separated from each other for a systematic analysis of the polarization dependent image dipole effects on the signal. Measured signals are fully explained by the Fabry-Perot like interference between the 
radiations from the GNP and from the image dipole induced at the flat gold surface, and by the finite size effects of the GNP.

We note that, in this study, flat surfaces to reflect the signal from the GNP are considered allowing a simple analysis of the detected signal. But, in real situations the sample may have a complex geometry and a more delicate treatment is required. To remove the complicate effects of the reflected light at the sample surface on the far-field detected signal, a confocallike spatial masking technique can be applied to cut out lights radiated other than from the GNP, when the distance of the GNP from the sample surface is bigger than the diffraction limit of the collection lens. Within a shorter distance range, one should carefully consider the reflections at the sample surface to correctly account for the radiation from the GNP.

\section{Problems and outlook}

The scattered light by GNP has same frequency of the local electric field, therefore to correctly designate the polarization vector of the source field it is crucial to detect only the light scattered from GNP excluding any other background light. In our works, the source fields, the standing waves and outgoing waves from a slit structure, with a symmetry to the detection position, do not send light by themselves to the detector. However, due to the imperfection of the sample structure and the rough sample surface may generate background lights. And also the glass tip shaft scatters light into the detector. To study the background light effects on the determined polarization vector direction, we changed the amount of the background light to the detector and measured the changes of the local polarization vector direction. The experimental setup is same as depicted in Fig. 14. The tip was positioned at one position $50 \mu \mathrm{m}$ away from the slit and the distance of the GNP from the sample surface was kept by few nm using a shear force feedback system. A spatial filter was placed in the image plane of the collection objective to cut out the background light other than from the GNP. The opening size of the spatial mask was increased allowing more background light to be detected. From the result in Fig. 15, in the vicinity of a flat gold surface, the polarization state of the scattered light from GNP is highly elliptical with the major axis of the ellipse standing vertically to the surface, along z-axis. With more background light, with wider opening the spatial mask, the major axis of the polarization ellipse deviates more from the vertical direction. The problem is that it is hardly possible to collect light only from the GNP for an arbitrary structured sample.

One possible way to make this local polarization vector detection method be applicable to an arbitrary situation including the self radiating samples is using the Stokes shifted light from nano-objects other than the GNP such as quantum dots and single molecules. For example, the absorption dipole moment of single molecules can be used to detemine the molecular orientation with carefully tailoring the excitation electric field (Betzig \& Chichester, 1993). There are remaining questions how to implement such an object to a tip in a controllable way of the position and the orientation. Loosing the coherence in the Stokes shifted light is another disadvantage in determining the temporal phase. Searching and designing of new types probes to extend the functionality of the polarization vector microscope are currently underway.

Even though the scattering from the glass tip shaft can be much less than from the GNP due to the dielectric constant, nevertheless it should not be ignored since the whole volume of 
the tip shaft is much bigger than of the GNP. By making the tip shaft thinner, scattering from it can be reduced down. But making the tip shaft too much thin may prevent a good performance of the feedback system in controlling the tip position in the near-field region. In addition, it is needed to study the energy transfer between the tapered glass tip and the metallic structures attached to the end of it, depending on the material and on the structural shape.

(a)
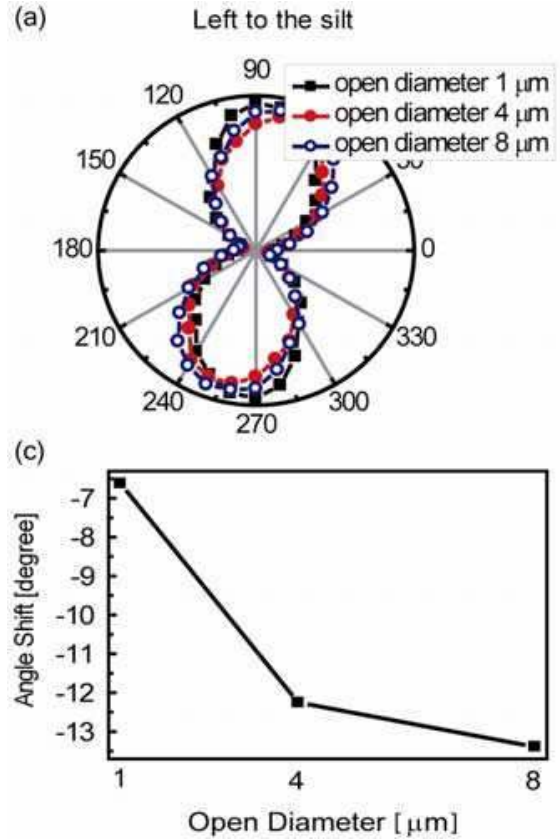

(b)

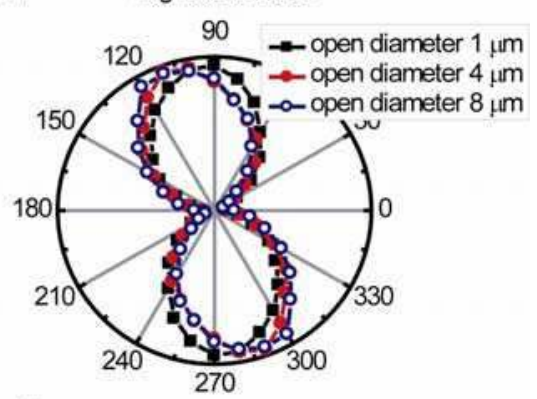

(d)

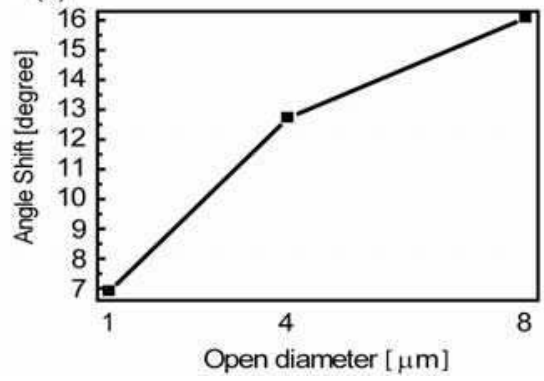

Fig. 16. Background light effect on the measured vector orientation. (a) Polar plot of the measured intensity. A gold nano-particle functionalized tip was located on a flat-metal region on the left-hand side of the slit about $50 \mu \mathrm{m}$ away from the slit position, where the vector field theoretically points towards the z-direction. When the iris opening of the spatial filter setup corresponds to a collection diameter of 8 microns, the experimental vector deviates from the $\mathrm{z}$ axis by minus 13.5 degrees. Closing the iris reduces this discrepancy to minus 7 degrees as shown in Figs. (a) and (c). (b) Moving the tip to the right-hand side of the slit and repeating the same experiments essentially gives the same results (Figs. (b) and (d)). From (Lee et al., 2007b). (c) 2007 Nature Publishing Group.

\section{Conclusion}

In this chapter, we demonstrate the local field polarization vector detection on nanoscale using the GNP attached tips as the local polarizer. This was enabled by means of a novel, scattering-type near-field microscopic technique combined with complete tipcharacterization. For a suitably small GNP with the diameter less than $100 \mathrm{~nm}$, the far-field scattering is dominated by the electric dipole radiation. Dipole radiation conserve its polarization state into the far-field region enabling characterization of the dipole moment 
induced at the GNP by measuring the far-field polarization state. And the dipole moment is determined by the local electric field via polarizability tensor of GNP. Therefore, by characterizing the polarizability tensor of GNP and the polarization state of far-field scattered light, the local electric field vector can be reconstructed. The polarizability tensor of the GNP is measured by two different scattering measurements-RPAE and RAE. And the polarization of the far-field scattered light is determined by applying RAE and Stokes measurements. By carefully considering the scattering shape of the GNP, the polarizability tensor, it is shown that the finally determined local polarization vectors are independent of the tip which confirms the reliability of our method. A full 3-dimensional polarization field mapping is demonstrated by detecting a focused radial polarization.

These results provide unprecedented images of light in nano-scale and demonstrate that the local field polarizatin vector mapping of light is indeed possible for nano-systems where spatially rapidly changing field orientations on a sub-wavelength scale are the rule rather than the exception and crucial for the functionality of novel nanophotonic devices. This method carry the potential for making the vector field mapping for nano-scale devices a common laboratory practice, instead of a conceptual one encountered only in theoretical realm, that can find wide applications in physics, engineering, chemistry and biology.

We also applied this method in studying the intricate surface effects when the scattering measruements are perfomed in the near-field. Supplementally, we discussed the limitations in our system and the possible ways to reconcile them to further improve the functionality of the system.

\section{References}

Ahn, J. S.; Kihm, H. W.; Kihm, J. E.; Kim, D. S. \& Lee, K. G. (2009). 3-dimensional local field polarization vector mapping of a focused radially polarized beam using gold nanoparticle functionalized tips. Opt. Express, 17, 4, (FEB 2009), (2280-2286), ISSN 1094-4087

Ahn, K. J.; Lee, K. G. \& Kim, D. S. (2008). Effect of dielectric interface on vector field mapping using gold nanoparticles as a local probe: Theory and experiment. Opt. Commun., 281, (AUG 2008), (4136-4141), ISSN 0030-4018

Betzig, E. \& Chichester, R. J. (1993). Single molecules observed by near-field scanning optical microscopy. Science, 262, (NOV 1993), (1422-1425), ISSN 0036-8075; online ISSN 1095-9203

Born, M. \& Wolf, E. (1999). Principles of Optics - $7^{\text {th }}$ ed., Cambridge University Press, ISBN-10: 0521642221; ISBN-13: 978-0521642224, Cambridge, England

Chew, H.; Wang, D. -S. \& Kerker, M. (1979). Elastic scattering of evanescent electromagnetic waves. Appl. Opt., 18, (AUG 1979), (2679-2687), ISSN 0003-6935

Cvitkovic, A.; Ocelic, N. \& Hillenbrand, R. (2007). Analytical model for quantitative prediction of material consrasts in scattering-type near-field optical microscopy. Opt. Express, 15, 14, (JUL 2007), (8550-8565), ISSN 1094-4087

Ellis, J. \& Dogariu, A. (2005). Optical Polarimetry of Random Fields. Phys. Rev. Lett., 95, (NOV 2005), (203905_1-4), ISSN 0031-9007 
Ganic, D.; Gan, X. \& Gu, M. (2003). Parametric study of three-dimensional near-field Mie scattering by dielectric particles. Opt. commun., 216, (FEB 2003), (1-10), ISSN 00304018

Jackson, J. D. (1998). Classical Electrodynamics, John Wiley \& Sons, Inc., ISBN-10: 047130932X; ISBN-13: 978-0471309321, New York

Kalkbrenner, T.; Ramstein, M.; Mlynek, J. \& Sandoghdar, V. (2001). A single gold particle as a probe for apertureless scanning near-field optical microscopy. J. Microsc., 202, (APR 2001), (72-76), ISSN 0022-2720

Keating, C. D.; Musick, M. D.; Keefe, M. H. \& Natan, M. J. (1999). Kinetics and Thermodynamics of $\mathrm{Au}$ Colloid Monolayer Self-Assembly: Undergraduate Experiments in Surface and Nanomaterials Chemistry. J. Chem. Ed. chem.wisc.edu., 76, (JUL 1999), (949), ISSN 0021-9584

Kihm, H. W.; Lee, K. G.; Kim, D. S.; Kang, J. H. \& Park, Q-Han (2008). Control of surface plasmon generation efficiency by slit-width tuning. Appl. Phys. Lett., 92, (FEB 2008), (051115), ISSN 0003-6951

Knoll, B. \& Keilmann, F. (2000). Enhanced dielectric contrast in scattering-type scanning near-field optical microscopy. Opt. Commun., 182, (AUG 2000), (321-328), ISSN 0030-4018

Kühn, S.; Håkanson, U.; Rogobete, L. \& Sandoghdar, V. (2006). Enhancement of SingleMolecule Fluorescence Using a Gold Nanoparticle as an Optical Nanoantenna. Phys. Rev. Lett., 97, (JUL 2006), (017402), ISSN 0031-9007

Lee, K. G.; Kihm, H. W.; Kihm, J. E.; Choi, W. J.; Kim, H.; Ropers, C.; Park, D. J.; Yoon, Y. C.; Choi, S. B.; Woo, D. H.; Kim, J.; Lee, B.; Park, Q. H.; Lienau, C. \& Kim, D. S. (2007a). Vector field microscopic imaging of light. Nature Photon., 1, (JAN 2007a), (53-56), ISSN 1749-4885

Lee, K. G.; Kihm, H. W.; Kihm, J. E.; Choi, W. J.; Kim, H.; Ropers, C.; Park, D. J.; Yoon, Y. C.; Choi, S. B.; Woo, D. H.; Kim, J.; Lee, B.; Park, Q. H.; Lienau, C. \& Kim, D. S. (2007b). On the concept of imaging nanoscale vector fields. Nature Photon., 1, (MAY 2007b), (243-244), ISSN 1749-4885

Lee, K. G.; Kihm, H. W.; Ahn, K. J.; Ahn, J. S.; Suh, Y. D.; Lienau, C. \& Kim, D. S. (2007c). Vector field mapping of local polarization using gold nanoparticle functionalized tips: independence of the tip shape. Opt. Express, 15, 23, (NOV 2007c), (1499315001), ISSN 1094-4087

Lee, K. G.; Ahn, K. J.; Kihm, H. W.; Ahn, J. S.; Kim, T. K.; Hong, S.; Kim, Z. H. \& Kim, D. S. (2008). Surface plasmon polariton detection discriminating the polarization reversal image dipole effects. Opt. Express, 16, 14, (JUL 2008), (10641-10649), ISSN 1094-4087

Lee, K. G.; Kihm, H. W. \& Kim, D. S. (2009). Measurement of the Polarizability Tensor of Gold-nanoparticle-functionalized Tips. J. Kor. Phys. Soc., (in press), ISSN 0374-4884

Martin, O. J. F.; Girard, C. \& Dereux, A. (1995). Generalized Field Propagator for Electromagnetic Scattering and Light Confinement. Phys. Rev. Lett., 74, (JAN 1995), (526-529), ISSN 0031-9007

Novotny, L. \& Hecht, B. (2006). Principles of Nano-Optics, Cambridge University Press, ISBN13: 9780521832243 ; ISBN-10: 0521832241, New York 
Raschke, M. B. \& Lienau, C. (2003). Apertureless near-field optical microscopy: Tip-sample coupling in elastic light scattering. Appl. Phys. Lett., 83, 24, (DEC 2003), (5089-5091), ISSN 0003-6951

Reather, H. (1988). Surface Polaritons on Smooth and Rough Surfaces and on. Gratings, SpringerVerlag, ISBN-10: 0387173633; ISBN-13: 978-0387173634, Berlin

Stokes, G. G. (1852). Trans. Cambridge Philos. Soc., 9, (399-416)

Youngworth, K. S. \& Brown, T. G. (2000). Focusing of high numerical aperture cylindricalvector beams. Opt. Express, 7, 2, (JUL 2000), (77-87), ISSN 1094-4087 


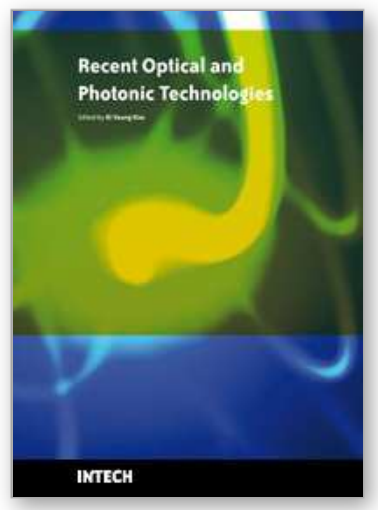

\author{
Recent Optical and Photonic Technologies \\ Edited by Ki Young Kim
}

ISBN 978-953-7619-71-8

Hard cover, 450 pages

Publisher InTech

Published online 01, January, 2010

Published in print edition January, 2010

Research and development in modern optical and photonic technologies have witnessed quite fast growing advancements in various fundamental and application areas due to availability of novel fabrication and measurement techniques, advanced numerical simulation tools and methods, as well as due to the increasing practical demands. The recent advancements have also been accompanied by the appearance of various interdisciplinary topics. The book attempts to put together state-of-the-art research and development in optical and photonic technologies. It consists of 21 chapters that focus on interesting four topics of photonic crystals (first 5 chapters), THz techniques and applications (next 7 chapters), nanoscale optical techniques and applications (next 5 chapters), and optical trapping and manipulation (last 4 chapters), in which a fundamental theory, numerical simulation techniques, measurement techniques and methods, and various application examples are considered. This book deals with recent and advanced research results and comprehensive reviews on optical and photonic technologies covering the aforementioned topics. I believe that the advanced techniques and research described here may also be applicable to other contemporary research areas in optical and photonic technologies. Thus, I hope the readers will be inspired to start or to improve further their own research and technologies and to expand potential applications. I would like to express my sincere gratitude to all the authors for their outstanding contributions to this book.

\title{
How to reference
}

In order to correctly reference this scholarly work, feel free to copy and paste the following:

Kwang Geol Lee and DaiSik Kim (2010). Local Electric Polarization Vector Detection, Recent Optical and Photonic Technologies, Ki Young Kim (Ed.), ISBN: 978-953-7619-71-8, InTech, Available from: http://www.intechopen.com/books/recent-optical-and-photonic-technologies/local-electric-polarization-vectordetection

\section{INTECH}

open science | open minds

\section{InTech Europe}

University Campus STeP Ri

Slavka Krautzeka 83/A

51000 Rijeka, Croatia

Phone: +385 (51) 770447

Fax: +385 (51) 686166

\section{InTech China}

Unit 405, Office Block, Hotel Equatorial Shanghai

No.65, Yan An Road (West), Shanghai, 200040, China 中国上海市延安西路65号上海国际贵都大饭店办公楼 405 单元

Phone: +86-21-62489820

Fax: +86-21-62489821 
www.intechopen.com 
(C) 2010 The Author(s). Licensee IntechOpen. This chapter is distributed under the terms of the Creative Commons Attribution-NonCommercialShareAlike-3.0 License, which permits use, distribution and reproduction for non-commercial purposes, provided the original is properly cited and derivative works building on this content are distributed under the same license. 